\title{
Principais determinantes nas intoxicações por fármacos na Cidade de Teresina-PI,
}

\section{Brasil}

\author{
Main determinants of drug poisoning in the City of Teresina-PI, Brazil \\ Principales determinantes de la intoxicación por drogas en la Ciudad de Teresina-PI, Brasil
}

\author{
Hercília Maria Lins Rolim \\ ORCID: https://orcid.org/0000-0003-4469-6858 \\ Universidade Federal do Piauí, Brasil \\ E-mail: hercilia.rolim@gmail.com \\ Elison Costa Holanda \\ ORCID: https://orcid.org/0000-0001-9130-7873 \\ Universidade Federal do Piauí, Brasil \\ E-mail: holandap2@outlook.com \\ José Arimatéa de Oliveira Nery Neto \\ ORCID: https://orcid.org/0000-0002-8239-1548 \\ Universidade Federal do Piauí, Brasil \\ E-mail: arineto@outlook.com \\ Andrew Guilherme Oliveira \\ ORCID: https://orcid.org/0000-0001-9056-9759 \\ Universidade Federal do Piauí, Brasil \\ E-mail: andreguilherme19@gmail.com \\ Leandro Soares da Silva Bessa \\ ORCID: https://orcid.org/0000-0001-7751-1421 \\ Universidade Federal do Piauí, Brasil \\ E-mail: leossb22@gmail.com \\ Evaldo Hipólito de Oliveira \\ ORCID: https://orcid.org/0000-0003-4180-012X \\ Universidade Federal do Piaú, Brasil \\ E-mail: evaldohipolito@gmail.com
}

\begin{abstract}
Resumo
Introdução: A ocorrência de óbitos por intoxicação com medicamentos tem sido considerada um dos agravos de saúde pública. Objetivo: Descrever as principais determinantes nas intoxicações por fármacos na cidade de Teresina-PI. Metodologia: Pesquisa ecológica, descritiva, quantitativa e de base populacional, com estudo de envenenamento por fármacos notificados na cidade de Teresina-PI, de 2010 a 2019 do Ministério da Saúde/SINAN. Foram coletados os seguintes dados: sexo, etnia, escolaridade, faixa etária, evolução clínica, classificação final, circunstância, critério de confirmação e o município de residência no Piauí. 2019.Resultados e discussão: No período analisado foi registrado 2.229 ocorrências, sendo preponderante a etnia parda $(41,36 \%)$, escolaridade com ensino médio completo $(12,43 \%)$, faixa etária de 20-39 (43,43\%) e sexo feminino (73,44\%). Já no tipo de exposição a aguda- crônica ocorreu em 1287 registros $(57,74 \%)$, e o critério de confirmação foi clínico (64,56\%). A circunstância mais frequente foi tentativa de suicídio $(71,02 \%)$ e o desfecho clinico final foi cura com ausência de dano $(59,22 \%)$. Conclusão: Constatou-se que na cidade de Teresina-PI ocorreu um aumento das notificações, esse fato pode ser atribuído ao padrão de consumo de fármacos. De modo geral, a Vigilância em Saúde deve priorizar a sensibilidade profissional para obter mais ações educativas, preventivas e intersetoriais, para assegurar o aprimoramento das políticas de vigilância sanitária.
\end{abstract}

Palavras-chave: Saúde pública; Epidemiologia; Envenenamento.

\begin{abstract}
Introduction: The occurrence of deaths due to drug intoxication has been considered one of the public health problems. Objective: To describe the main determinants of drug poisoning in the city of Teresina-PI. Methodology: Ecological, descriptive, quantitative and population-based research, with a study of poisoning by drugs reported in the city of Teresina-PI, from 2010 to 2019 by the Ministry of Health/SINAN. The following data were collected: gender, ethnicity, education, age group, clinical evolution, final classification, circumstance, confirmation criteria and the municipality of residence in Piauí. 2019. Results and discussion: In the period analyzed, 2,229 occurrences were recorded, with the predominant ethnicity $(41.36 \%)$, education with complete high school (12.43\%), age group 20-39 (43.43\%) and female (73.44\%). As for the type of exposure to acute-chronic, it occurred in 1287 records $(57.74 \%)$, and the criterion for confirmation was clinical $(64.56 \%)$. The most frequent circumstance was attempted suicide $(71.02 \%)$ and the final clinical outcome was cure with no damage (59.22\%). Conclusion: It was found that in the city of Teresina-
\end{abstract}


PI there was an increase in notifications, this fact can be attributed to the pattern of drug consumption. In general, Health Surveillance must prioritize professional sensitivity to obtain more educational, preventive and intersectoral actions to ensure the improvement of health surveillance policies.

Keywords: Public health; Epidemiology; Poisoning.

\section{Resumen}

Introducción: La ocurrencia de muertes por intoxicación por drogas ha sido considerada uno de los problemas de salud pública. Objetivo: Describir los principales determinantes de la intoxicación por drogas en la ciudad de Teresina-PI. Metodología: Investigación ecológica, descriptiva, cuantitativa y poblacional, con estudio de intoxicaciones por drogas reportadas en la ciudad de Teresina-PI, de 2010 a 2019 por el Ministerio de Salud / SINAN. Se recogieron los siguientes datos: sexo, etnia, escolaridad, grupo de edad, evolución clínica, clasificación final, circunstancia, criterios de confirmación y municipio de residencia en Piauí. 2019. Resultados y discusión: En el período analizado se registraron 2.229 ocurrencias, con la etnia predominante $(41,36 \%)$, educación con bachillerato completo (12,43\%), grupo etario $20-39(43,43 \%)$ y femenino $(73,44 \%)$. En cuanto al tipo de exposición a aguda-crónica, ocurrió en 1287 registros $(57,74 \%)$, y el criterio de confirmación fue clínico $(64,56 \%)$. La circunstancia más frecuente fue el intento de suicidio $(71,02 \%)$ y el resultado clínico final fue la curación sin daño $(59,22 \%)$. Conclusión: Se encontró que en la ciudad de Teresina-PI hubo un aumento en las notificaciones, este hecho se puede atribuir al patrón de consumo de drogas. En general, la Vigilancia en Salud debe priorizar la sensibilidad profesional para obtener acciones más educativas, preventivas e intersectoriales que aseguren la mejora de las políticas de vigilancia en salud.

Palabras clave: Salud pública; Epidemiología; Envenenamiento.

\section{Introdução}

Os fármacos, por serem de fácil acesso, são a principal causa de intoxicação tornando se assim um grave problema, devido ao uso desregulado, gerando inúmeros óbitos e internações hospitalares (Silva\& Alvarez,2018). Atualmente cerca de 350 mil casos de mortes por envenenamento por drogas ocorrem no mundo, sendo pelo menos $10 \%$ em inferiores a idade de 15 (Lopes et al., 2019). Dados do Relatório Anual da Associação Americana dos Centros de Controle de Intoxicações (AAPCC) demonstram que, dentre as 25 categorias de substâncias que mais aumentaram nos atendimentos realizados nos anos de 2000 a 2012, 20 são medicamentos, sendo os principais os analgésicos, hipnóticos e ansiolíticos (Costa \& Alonzo, 2015).

Conforme Silva \& Álvares (2019) o Brasil ocupa a quinta posição na lista mundial de consumo de fármacos, além de situar-se em primeiro na América Latina. E ainda segundo a Associação Brasileira das Indústrias Farmacêuticas (Abifarma), o uso irracional de fármacos é a opção de 80 milhões de pessoas o que culmina a intoxicação medicamentosa. Dados registrados apontam que, entre 2000 e 2012, foram notificados mais de 300 mil registros de envenenamento por fármacos e, desses 1.102 (0,3\% dos casos) evoluíram para o óbito (Santos \& Boing, 2018).

$\mathrm{Na}$ intoxicação medicamentosa o organismo sofre lesões ou danos provocados por quantidade excessiva ou por efeito cumulativo de substâncias que ministradas normalmente em doses apropriadas seriam benéficas a saúde (Souto et al., 2012).Esse tipo de intoxicação pode ser classificada em agudas ou crônicas, onde cada droga apresenta suas particularidades num quadro de sinais e sintomas (Gonlçalves et al., 2017). Isso fez com que incidentes com medicamentos tenham chamado a atenção de profissionais, instituições e autoridades sanitárias, devido ao fato de contribuírem para a morbidade e da estada hospitalar (Santos \& Boing, 2018).

Nesse sentido, os números crescentes de intoxicação medicamentosas geram mudanças desfavoráveis, uma vez que contribuem para um aumento da demanda dos serviços e nos custos financeiros para o poder público (Ranger; Francelino, 2018). Além de ser um importante guia para a gestão de recursos destinados ao planejamento e a implementação de ações que previnam a ocorrência desse agravo (Teles et al., 2013).

Apesar da existência de dados epidemiológicos sobre intoxicações em nível nacional e regional, a realidade em municípios ainda é desconhecida, em razão da vulnerabilidade da população a intoxicações a exposição a diferentes substâncias que podem ser tóxicas (Oliveira \& Suchara, 2014). O estudo descreve as principais determinantes nas intoxicações por fármacos na cidade de Teresina-PI. 


\section{Metodologia}

Para realização de estudos científicos é necessário métodos e técnicas que melhor articulem o andamento da pesquisa, contudo existem formas documentais em institutos de pesquisa, órgãos públicos e departamentos de registro e estatística (Oliveira et al., 2019).

Esta é uma pesquisa ecológica, de caráter descritivo, de abordagem quantitativa e de base populacional, utilizando-se dados secundários epidemiológicos dos casos por envenenamento por fármacos notificados na cidade de Teresina-PI, no intervalo de 2010 a 2019. Esse período de tempo foi determinado por abranger o intervalo em que os dados se encontram completos no Sistema de Informação de Agravos de Notificação (SINAN). Foram usados dados pertencentes a um banco de dados genuíno e de livre acesso, o DATASUS (Departamento de Informática do Sistema Único de Saúde), presentes no SINAN. O SINAN consiste em uma ferramenta com abrangência em todo território nacional e de caráter universal, que auxilia na vigilância epidemiológica, apoiando na tomada de decisões (Emmericket al, 2014). As informações estão na seção de informação de saúde (TABNET), na opção epidemiologia e morbidades.

O programa Microsoftæ Excel 2019 foi utilizado para organização dos dados. Dentre as diversas variáveis disponibilizadas pelo SINAN levou-se em consideração neste estudo o sexo, etnia, escolaridade, faixa etária, evolução clínica, classificação final, circunstância, critério de confirmação e o município de residência no Piauí. Após colocados em gráficos e mapas, com a prevalência por município de infecção obtido através do programa Tabwin, do site DATASUS.

\section{Resultados e Discussão}

Através da análise espacial georreferenciada na Figura 1 foi possível avaliar a distribuição dos registros no Piauí de 2010-2014, com 1543 ocorrências, onde o maior número de notificações ocorreu na cidade de Teresina (n=573;37,15\%), seguido de Picos ( $\mathrm{n}=375 ; 24,3 \%)$ e Parnaíba ( $\mathrm{n}=213 ; 13,8 \%)$. Já na figura 2 a distribuição no Piauí de 2015-2019, com 4465 registros, onde o maior número de notificações ocorreu na cidade de Teresina ( $\mathrm{n}=1706 ; 38,2 \%)$, seguido de Picos $(\mathrm{n}=904 ; 20,2 \%)$ e Floriano (n=633;14,1\%).Desta forma pode-se observar uma distribuição heterogênea do agravo (Figura 2). 
Figura 1 - Análise espacial georreferenciada da distribuição dos registros de intoxicações por fármacos no Piauí de 2010 -2014.



Fonte: Ministério da Saúde/SVS - SINAN Net. Dados exportados em janeiro de 2021.

Figura 2 - Análise espacial georreferenciada da distribuição dos registros por intoxicações por fármacos no Piauí de 2015-2019.

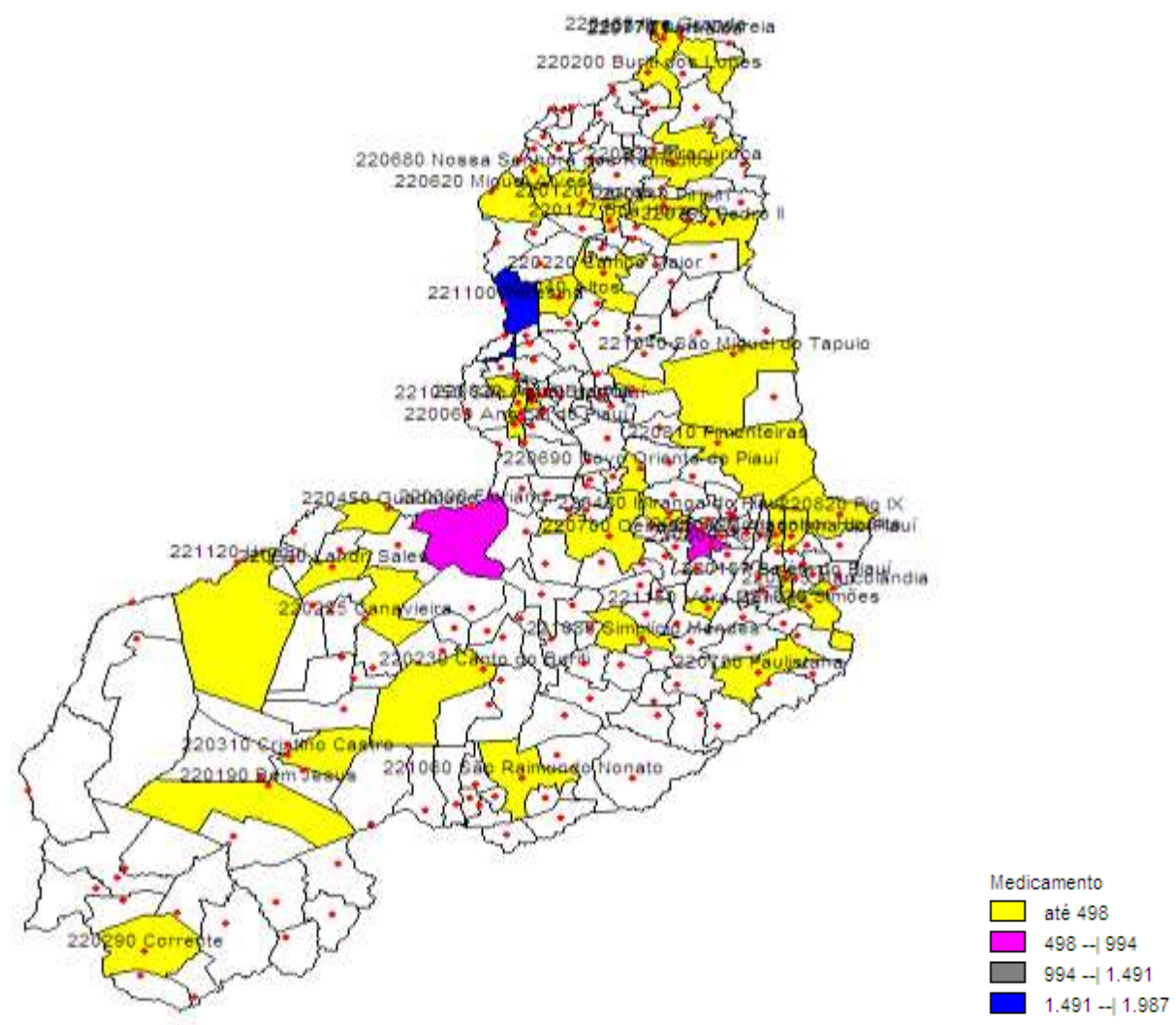

Fonte: Ministério da Saúde/SVS - SINAN Net. Dados exportados em janeiro de 2021. 
A extensa variedade de medicamentos favorece problemas relacionados a estes produtos, representando um desafio ao sistema de saúde no Brasil, assim como nas grandes cidades do Piaui. Outros fatores que contribuem significativamente para esse quadro são: a frágil regulação da publicidade acerca do medicamento, a facilidade na aquisição de fármacos sob prescrição médica, a deficiência de legislação específica sobre embalagens seguras, escassas iniciativas de desenvolvimento da atenção farmacêutica, e o padrão do consumo de fármacos na população, caracterizado pela automedicação, polifarmácia, uso indevido e indiscriminado, principalmente de psicotrópicos e antibióticos (Nobrega et al.,2015).

Entre 2010 a 2018 observou-se um aumento das notificações de envenenamento por fármacos por ano na cidade de Teresina-PI, com uma pequena redução em 2019. Foram confirmados 2.229 casos, perfazendo uma média anual de 222,9 atendimentos. Como demonstrado no gráfico 1, o ano que apresentou o maior registro foi em 2018, totalizando 429 notificações $(19,5 \%)$, seguido de 2017 ( $n=339 ; 15,21 \%)$. Por outra perspectiva, os menores valores corresponderam a $2010(n=53 ; 2,38 \%)$ e $2011(\mathrm{n}=78 ; 3,5 \%)$, indicando um provável aumento de casos, entretanto, sem descartar necessidades de melhorias nas notificações.

Estes achados estão em conformidade com estudo de Lopes et al., (2019) nesse mesmo município entre 2010-2017 em crianças, com maior registro em 2017 (n=60;17,5\%). O crescimento no número absoluto de registros nessa pesquisa se deve ao maior grau de conhecimento dos profissionais das instituições de saúde quanto ao oferecimento de atendimento especializado, tanto da parte clínica quanto laboratorial (Costa et al., 2015). Paralelo a isso, há maior oferta de medicamentos de venda livre (Magalhães et al., 2013). O aumento progressivo de intoxicações nesse estudo relacionadas com a exposição a medicamentos, chama a atenção para um problema capaz de gerar danos aos indivíduos, bem como onerar os sistemas de saúde (Teles et al., 2013).

Gráfico 1 - Registro por Agente Tóxico-fármaco segundo Ano $1^{\circ}$ Sintoma (s), entre 2010-2019, na cidade de Teresina-Piauí.

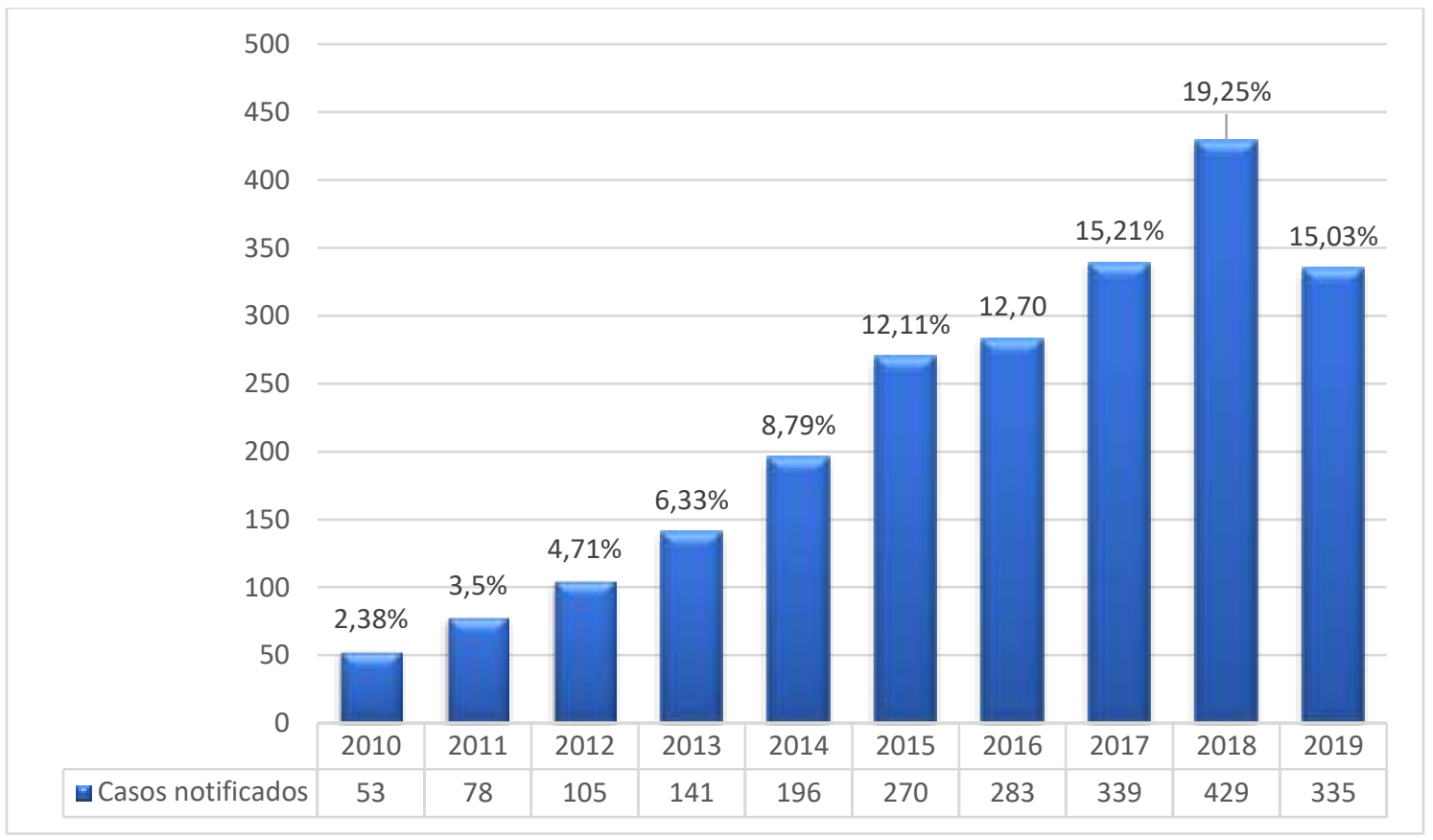

Fonte: Ministério da Saúde/SVS - SINAN Net. Dados exportados em janeiro de 2021. Os dados de 2017 a 2019 estão sujeitos à revisão, segundo TABNET.

Quanto à distribuição por sexo, na tabela 01, verificou-se o predomínio do sexo feminino (73,44\%). Estes dados foram semelhantes aos descritos por um hospital municipal de Itapira-SP, entre janeiro a dezembro de 2012, no qual $61 \%$ dos casos ocorreram em mulheres entre 20 a 39 anos (Germano \& Alonzo, 2015). Segundo Nunes et al., (2017) este fato justifica-se 
já que as mulheres têm maior preocupação com a saúde do que os homens, devido a questões fisiológicas que lhes atribuem uma maior necessidade de utilização de medicamentos, dessa forma, procuram mais os hospitais e consequentemente tornando-as mais expostas ao risco de intoxicação. Já Paula et al., (2012) atribuem tal fato à um maior índice de longevidade nesse sexo.

Tabela 1 - Distribuição das variáveis epidemiológicas referente ao Agente Tóxico-fármaco segundo Ano $1^{\circ}$ Sintoma (s), entre 2010-2019, na cidade de Teresina-Piauí.

\begin{tabular}{|c|c|c|c|}
\hline & Variáveis & $\mathrm{N}$ & $\%$ \\
\hline \multicolumn{4}{|l|}{ Gênero } \\
\hline & Masculino & 592 & 26,56 \\
\hline & Feminino & 1637 & 73,44 \\
\hline \multicolumn{4}{|c|}{ Faixa etária } \\
\hline & Branco/Ignorado & 0 & 0 \\
\hline & $<1$ ano & 17 & 0,76 \\
\hline & $1-4$ & 256 & 11,49 \\
\hline & $5-9$ & 101 & 4,53 \\
\hline & $10-14$ & 144 & 6,46 \\
\hline & $15-19$ & 362 & 16,24 \\
\hline & $20-39$ & 968 & 43,43 \\
\hline & $40-59$ & 314 & 14,09 \\
\hline & $60-64$ & 27 & 1,21 \\
\hline & $65-69$ & 12 & 0,54 \\
\hline & $70-79$ & 17 & 0,76 \\
\hline & A partir de 80 anos & 11 & 0,49 \\
\hline \multicolumn{4}{|l|}{ Etnia } \\
\hline & Ignorado/ Branco & 838 & 37,60 \\
\hline & Branca & 294 & 13,19 \\
\hline & Preta & 153 & 6,86 \\
\hline & Amarela & 20 & 0,90 \\
\hline & Parda & 922 & 41,36 \\
\hline & Indígena & 2 & 0,09 \\
\hline \multicolumn{4}{|c|}{ Escolaridade } \\
\hline & Ignorado/ Branco & 769 & 34,50 \\
\hline & Analfabeto & 30 & 1,35 \\
\hline & $1^{\mathrm{a}}$ a $4^{\mathrm{a}}$ serie incompleto do $\mathrm{EF}$ & 93 & 4,17 \\
\hline & $4^{\mathrm{a}}$ série completa do EF & 34 & 1,53 \\
\hline & $5^{\mathrm{a}}$ a $8^{\mathrm{a}}$ serie incompleta do EF & 223 & 10,0 \\
\hline & Ensino fundamental completo & 79 & 3,54 \\
\hline & Ensino médio incompleto & 204 & 9,15 \\
\hline & Ensino médio completo & 277 & 12,43 \\
\hline & Educação superior incompleto & 126 & 5,65 \\
\hline
\end{tabular}




\begin{tabular}{llll}
\hline & Educação superior completo & 66 & 2,96 \\
\hline & Não se aplica & 328 & 14,72 \\
\hline Critério de confirmação & & & \\
\cline { 2 - 3 } & Ignorado/Branco & 97 & 4,35 \\
\hline Clinico -Laboratorial & 27 & 1,21 \\
\hline Clinico- Epidemiológico & 666 & 29,88 \\
\hline Clínico & 1439 & 64,56 \\
\hline
\end{tabular}

Legenda: EF (Ensino Fundamental); EM (Ensino Médio); ES (Ensino Superior).

Fonte: Ministério da Saúde/SVS - -SINAN Net. Dados exportados em janeiro de 2021. Os dados de 2017 a 2019 estão sujeitos à revisão, segundo TABNET.

Nessa pesquisa a idade que apresentou um maior registro, correspondeu ao que abrange a faixa etária de 20-39 (43,43\%; n=968) e dos 15 aos 19 anos (16,24\%; n=362), como evidenciado na Tabela 1. Fato esse que se observa maior concentração de casos na população economicamente ativa. Resultados semelhantes são reportados por Monte et al., (2016) no Piauí entre 2007 a 2012, em predomínio a faixa etária de 20-29(27,3\%) e 30-39(36,4\%) anos. Para Ficher \& Vansan (2008) o consumo de drogas psicotrópicas vem sendo consumida cada vez mais precoce pela população de adolescentes e adultos jovens e há uma associação importante entre dependência de drogas e morbidade psiquiátrica, principalmente depressão, que levam essa população ao uso dessas substancias e a comportamentos suicidas. Para Batista et al., (2017) a medida que a faixa etária entra em período na qual o indivíduo vai se tornando mais produtivo do ponto de vista trabalhista, expõe esse grupo etário a essa circunstância. Isso chama a atenção para o impacto socioeconômico de tais agravos, considerando-se que são idades correspondentes ao auge da vida produtiva do ser (Maia et al., 2019).

Em relação ao perfil de etnia na cidade de Teresina-PI houve destaque para pardos (41,36\%) e brancos (13,19\%). Conforme dados apontados pelo IBGE (2010) 61,8\% eram da população dessa cidade era de etnia parda, fato esse que é considerada uma característica da população da cidade em estudo.

A escolaridade é um fator que pode influenciar nestes registros. Neste estudo, a escolaridade foi preponderante no ensino médio completo $(n=277 ; 12,43 \%)$ e $5^{\circ}$ a $8^{\circ}$ ano do ensino fundamental $(n=223 ; 10,0 \%)$. A desinformação e o desconhecimento, por parte da população, sobre o uso correto de medicamentos, pode acarretar risco de intoxicações (Santana et al.,2019). Ademais quando se trata de possíveis projetos de prevenção e sobre a utilização adequada de fármacos , a falta de informação sobre escolaridade implica de maneira negativa, pois sem saber o público alvo é difícil acertar a didática que realmente apresente resultados satisfatórios (Palhano, 2020).

Tendo como base os indicadores operacionais de vigilância, pôde-se observar ainda na Tabela 01 que no critério de confirmação 64,56\% (n=1439) foram de diagnóstico clínico. Este diagnóstico nas intoxicações ocorre através do exame físico detalhado com avaliação dos sinais vitais, alterações metabólicas, neurológicas e comportamentais. Para o profissional habituado a análise das manifestações clínicas é o suficiente para o diagnóstico através da história clínica bem conduzida dependendo das circunstâncias da exposição e no grau de confiabilidade da informação prestada ao serviço de saúde (Queiroz et al.,2011).

Destaca-se neste estudo o baixo percentual de diagnóstico clínico-laboratorial $(1,21 \% ; n=27)$. Fato esse destacado na literatura como sendo relacionado pelas dificuldades no contexto de agravos relacionados ao trabalho, diagnósticos equivocados, escassez de testes específicos e de laboratórios para monitoramento biológico (Brasil, 2018). Em função disso é de extrema importância estimular o desenvolvimento de métodos mais eficazes e seguros e orientar o profissional da saúde na correta identificação e tratamento de indivíduos acometidos por reações adversas provocadas pela exposição aguda e crônica (Vinhal \& Soares,2018). 
Tabela 2 - Notificações de casos confirmados por agente tóxico-fármaco Ano $1^{\circ}$ Sintoma (s), de evolução clinica dos pacientes(s) segundo tipo de exposição no período de 2010-2019, na cidade de Teresina-Piauí.

Evolução clínica

Tipo de exposição

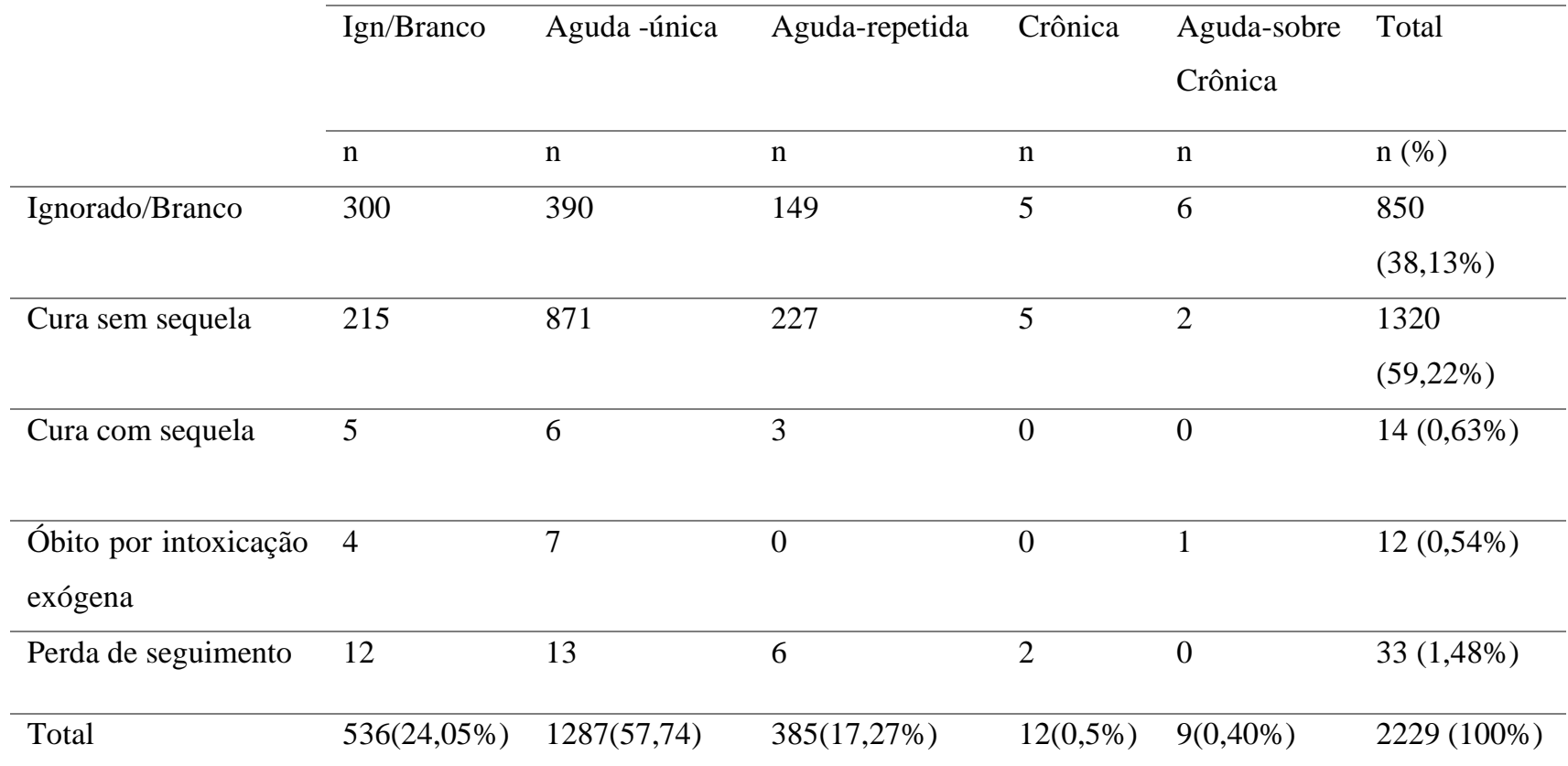

Fonte: Ministério da Saúde/SVS - SINAN Net. Dados exportados em janeiro de 2021. Os dados de 2017 a 2019 estão sujeitos à revisão, segundo TABNET.

No desfecho clinico dos pacientes segundo tipo de exposição, a cura sem agravos apresenta 1320(59,22\%) atendimentos. Estes resultados vão de encontro aos de Ramos et al., (2017), em São Paulo, na qual a cura sem agravos foi o critério de evolução da intoxicação mais frequente, com 99,6\%. Embora neste estudo a perda de seguimento (n=33; 1,48\%\%), cura com sequela $(n=14 ; 0,63 \%)$, morte por intoxicação exógena $(n=12,0,54 \%)$ sejam evidenciadas em baixos índices, deve-se considerar que as intoxicações são agressões ao equilíbrio homeostático e se constituem em questão de dano sanitário (Ramos et al.,2017).

Quanto ao tipo de exposição, destaca-se as exposições aguda- única com 61,8\%(n=1072) e aguda- repetida 14,6\% $(n=254)$. A intoxicação aguda é caracterizada por sinais que surgem rapidamente, cerca de algumas horas após a exposição excessiva a estes produtos. Os sinais e sintomas são claros, o que facilita o diagnóstico (Vinhal \& Soares, 2018).Porém, segundo Silva \& Costa (2018) em estudo de intoxicação exógenas em Santa Catarina a morbimortalidade torna-se significativa em pessoas que têm exposições com múltiplas drogas em situações agudas de baixas doses ao longo da vida, visto que ocasiona em efeitos crônicos, acarretando danos irreversíveis, do tipo paralisias e neoplasias.

Entretanto, 38,13\%(n=850) das notificações estavam em branco/ignoradas no desfecho clínico final e 536 (24,05\%) para o critério de exposição. As subnotificações são evidentes e, portanto, um problema a ser sanado quando se pensa em diminuir intoxicações agudas e crônicas provocadas fármacos. Falta de especialistas na área de vigilância epidemiológica e ambiental pode estar contribuindo para a subnotificação na cidade de Teresina-PI. Portanto, é de fundamental importância que os registros sejam realizados de forma correta no ato do diagnóstico dos pacientes, principalmente nos serviços de urgências (Matos et al., 2013). 
Gráfico 2 - Registro por agente tóxico-fármaco por circunstância entre 2010-2019, na Cidade de Teresina-Piauí.

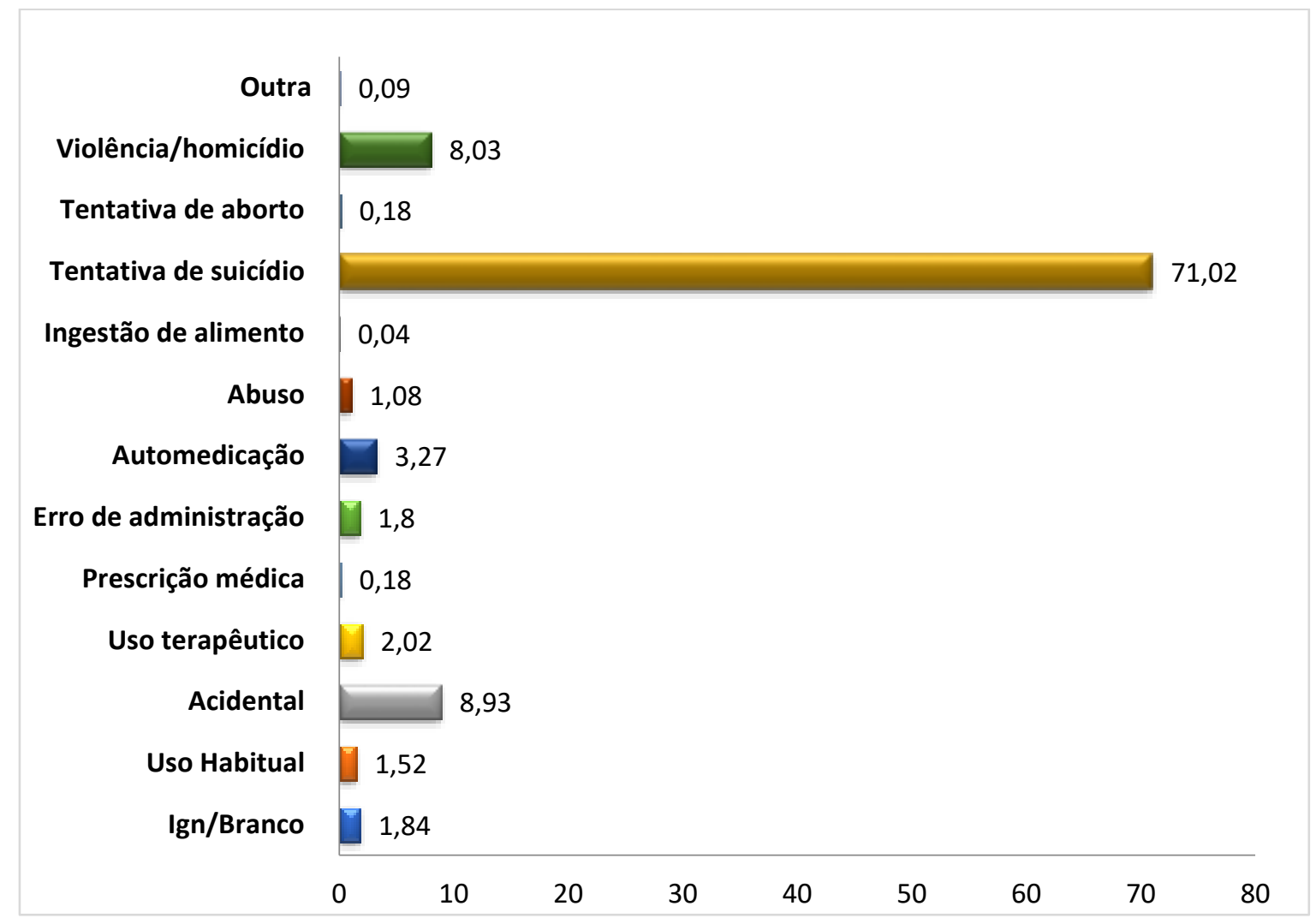

Fonte: Ministério da Saúde/SVS - SINAN Net. Dados exportados em em janeiro de 2021. Os dados de 2017 a 2019 estão sujeitos à revisão, segundo TABNET.

No Gráfico 2, 71,02\% ( $\mathrm{n}=1583)$ dos casos ocorreram devido a tentativa de suicídio, 8,93\% ( $\mathrm{n}=199)$ acidental e $3,27 \%(\mathrm{n}=73)$ automedicação. As menores incidências corresponderam a ingestão de alimentos $(\mathrm{n}=1 ; 0,04 \%)$, outras causas ( $\mathrm{n}=2$; $0,09 \%)$ e prescrição médica $(\mathrm{n}=4 ; 0,18 \%)$ junto à aborto $(\mathrm{n}=4 ; 0,18 \%)$. Esses dados estão de acordo com Silva (2018) que nos anos entre 2015-2016 com 18.729 registros na tentativa de suicídio por fármacos no Brasil. De forma semelhante, Costa (2015) detectaram que a tentativa de suicídio por medicamentos em Campinas entre 1998 a 2011 foi 45\% dos registrados. Entre as condições que mais favorecem a essa exposição indiscriminada da população aos medicamentos se deve ao acesso de antipsicóticos e anticonvulsivantes, apontadas a falta de controle da legislação federal sobre a produção, distribuição e comercialização (Viana, 2009).

Segundo Medeiros et al., (2014) as circunstâncias produtoras de estresse podem explicar o motivo da elevada proporção de tentativas de suicídio por medicamentos encontrada neste estudo, entre esses fatores, destacam-se o desemprego, a pobreza, problemas no trabalho, o uso abusivo de álcool e os distúrbios psíquicos. Para Vierira \& Caveião (2016) deve-se considerar que a tentativa de suicídio é elevada decorrente de psicofármacos entre os medicamentos, de modo que aprimorar as ferramentas legislativas de controle sobre prescrição e dispensação destes medicamentos poderia ser uma medida efetiva para reduzir as estatísticas sobre intoxicações medicamentosas, intencionais ou não.

\section{Conclusão}

Constatou-se que ocorreu uma maior predominância na etnia parda, escolaridade com ensino médio completo e $5^{\circ}$ a $8^{\circ}$ ano do ensino fundamental, faixa etária de 20-39 e sexo feminino. O tipo de exposição foi agudo-única, e o critério de 
confirmação foi clínico. A circunstância frequente foi tentativa de suicídio, sendo o desfecho clinico final foi a cura sem sequela. Ainda, notou-se o aumento do agente tóxico/ano no município de Teresina, atribuído a crescente desordem na comercialização de fármacos.

A importância de se conhecer o perfil epidemiológico das intoxicações predispõe da necessidade de alcançar chances de evitar essas intoxicações. Os farmacêuticos possibilitam que a sociedade receba orientações sobre os perigos da ingestão acidental de medicamentos, podendo, desta forma, evitando causar reações indesejadas ou intoxicação pelo uso indiscriminado. É imprescindível mais estudos desta ordem para que se possa acompanhar as principais determinantes nos envenenamentos por fármacos na cidade de Teresina-Piauí, com o intuito de estabelecer estratégias que previnam novos casos e de reincidências.

\section{Referências}

Augusto, S. S. D. (2010). Intoxicação medicamentosa: caracterização dos casos mortais. Dissertação (Mestrado). Faculdade de Medicina da Universidade de Coimbra. Mar. Disponível em: https://estudogeral.uc.pt/handle/10316/81239

Batista, L. A., Sousa, M. D. R., Rocha, R. J., \& Lacerda, E. M. C. B. (2017). Perfil epidemiológico dos casos de intoxicação notificados no Estado do Maranhão. Rev. Investig, Bioméd. São Luís, 9(2): 129-137.

Brasil. (2018). Intoxicações exógenas relacionadas ao trabalho no Brasil, 2007-2016. Secretaria de Vigilância em Saúde. Ministério da Saúde. Boletim Epidemiológico. 49 (58): Dez.

Brasil. (2014). Ministério da Saúde. Departamento de Informática do Sistema Único de Saúde - DATASUS. Indicadores e Dados Básicos (IDB) 2008. Brasília, DF: MS.

Costa, A. O. (2015). Atendimentos registrados no Centro de Controle de Intoxicações de Campinas: análise do período de 1998 a 2011 . 206 p. Dissertação (Mestrado) - Universidade Estadual de Campinas, Faculdade de Ciências Médicas, Campinas, SP. Disponível em: http://repositorio.unicamp.br/jspui/handle/REPOSIP/312705

Costa, A. O. C., \& Alonzo, H. G. A. (2015). Casos de exposições e Intoxicações por medicamentos registrados em um Centro de Controle de intoxicações do interior do Estado de São Paulo. Rev. Bras. Pesq. Saúde, Vitória, 17(2): 52-60, abr.-jun.

Emmerick, I. C. M., Campos, M. R., Schramm, J. M. D. A., Silva, R. S. D., \& Costa, M. D. F. D. S. (2014). Estimativas corrigidas de casos de meningite, Brasil 2008-2009. Epidemiologia e Serviços de Saúde, 23, 215-226

Ficher, A. M. F. T., \& Vansan, G. A. (2008). Tentativas de suicídio em jovens: aspectos epidemiológicos dos casos atendidos no setor de urgências psiquiátricas de um hospital geral universitário entre 1988 e 2004. Estud. psicol., Campinas, 25 (3): 361-374, set.

Germano, L. C., \& Alonzo. H. G. (2015). Intoxicações e reações adversas a medicamentos perfil local de subnotificação nos sistemas de informação em saúde. Eletronic Journal of Pharmacy, 12 (4): 32-44.

Gonçalves, C. A., Santos, V. A., Sarturi, L.; \& Terra, A. T. Jr. (2017). Intoxicação medicamentosa: relacionada ao uso indiscriminado de medicamentos. Revista Científica da Faculdade de Educação e Meio Ambiente, 8 (1): 135-143, jan.-jun.

IBGE (2010). Instituto Brasileiro de Geografia e Estatística. Cidades@: Teresina[Internet]. Rio de Janeiro: IBGE. Disponível em: https://cidades.ibge.gov.br/brasil/pi/teresina/pesquisa/23/25124. Acesso em: 15 de junho de 2020.

Lopes, D. S., Bispo, D. C., Carvalho, F. S., Macêdo, K. P. C., Freitas, R. L. Oliveira, E. H., \& Sousa. J. A. (2019). Intoxicações por medicamentos em pacientes pediátricos: análise na cidade de Teresina, Piauí. Brazilian Journal of Surgery and Clinical Research - BJSCR. 27 (1): 33-36, Jun.-Ago,.

Maestri, K. C. Y. O.; Viana, A. F. S., Lima, A. M. C.; Aguiar, D. X. G. B., Nogueira, A. M., Pereira, P. M. P., Glaser, A., \& Maestri, R. P. (2016). Intoxicações exógenas no município de Santarém- Parános anos de 2009 A 2013. Revista da Universidade Vale do Rio Verde, Três Corações, 14 (1): 647-656, jan.-jul..

Maior, M. C. L. S., Castro, C. G. S. O., \& Andrade, C. L. T. (2017). Internações por intoxicações medicamentosas em crianças menores de cinco anos no Brasil, 2003-2012. Epidemiol. Serv. Saúde, Brasília, 26(4):771-782, out - dez.

Maia, S. S., Souza, V. S., Souza, E. D., \& Faustino, T. N. (2019). Anos potenciais de vida perdidos por intoxicação exógena no Brasil no período de 2007 a 2017. Rev. Enferm Contemp.;8(2):135-142. Doi: 10.17267/2317-3378rec.v8i2.2447.

Magalhães, J. V., Monte, B. S., Santos, M. B., Rocha, L. P. V., \& Mendes ,C. M. M. (2013) Caracterização das intoxicações medicamentosas registradas no centro de informações toxicológicas do Piauí no período de 2007 a 2012. Revista de Pesquisa Cuidado é Fundamental, 5 (6): 55-63,dez. DOI: 10.9789/21755361.2013v5n6Esp2p55.

Medeiros, M. N. C., Medeiros, M. C., \& Silva, M. B. A. (2014). Intoxicação aguda por agrotóxicos anticolinesterásicos na cidade do Recife, Pernambuco, 20072010. Epidemiol. Serv. Saúde, Brasília, 23(3): 509-518,jul- set.

Monte, B. S. M., Nunes, M. S. T., Nunes, M. D. S., \& Mendes, C. M. M. (2016). Estudo epidemiológico das intoxicações por medicamentos registrados pelo centro de informações toxicológicas do Piauí: 2007 a 2012. R. Interd. 9( 3): 96-104, jul. ago. set.

Matos, A. S. (2013). Análise das intoxicações exógenas por agrotóxicos no Brasil, entre 2007 a 2012. Tese (TCC). Universidade de Brasília - UnB. Faculdade de Ceilândia -. Brasília. Disponível em: https://bdm.unb.br/handle/10483/6913 
Nóbrega, H. O. S.\& Costa, A. M. P., Saulo Rios Mariz, S. R., Fook,S. (2015).M. Intoxicações por medicamentos: uma revisão sistemática com abordagem nas síndromes tóxicas. Revista saúde e ciência On line,; 4(2): 109-119.

Nunes, C. R. M., Alencar, G. O., Bezerra,C. A., Barreto, M. F. R., \& Saraiva, E. M. S. (2017). Panoramas das intoxicações por medicamentos no Brasil. Rev. eciência, 5(2): 98-103.

Oliveira, F. F. S., \& Suchara. A. E. (2014). Perfil epidemiológico das intoxicações exógenas em crianças e adolescentes em município do Mato Grosso. Rev. paul. pediatr. 32 (4).

Oliveira, E. H., Silveira, J. A. V. da, Sampaio, S. S. de C., Verde, R. M. C. L., Soares, L. F., \& Costa, S. C. R. (2020). Análise dos casos notificados de sífilis na gestação no estado da Paraíba, Brasil. Research, Society and Development, 9(1), e179911900. https://doi.org/10.33448/rsd-v9i1.1900

Queiroz, C. M. S., Fonseca, S. A., \& Silva, A. C. P. (2011). Análise de casos de intoxicação por substâncias químicas e medicamentos no hospital geral do estado de Alagoas. Infarma, 24 (5).

Santana, V. T. P., Sucharab, E. A., Carreto, R., \& Duarte, P. M. (2019). Perfil das Intoxicações Medicamentosas Notificadas ao SINAN no Município de Primavera do Leste - MT, Entre os Anos de 2007 a 2014. Ensaios e Ciênc. 23( 3): 230-237.

Santos, G. A. S., \& Boing, A.C. (2018). Mortalidade e internações hospitalares por intoxicações e reações adversas a medicamentos no Brasil: análise de 2000 a 2014. Cad. Saúde Pública,34 (6): 25.

Silva, E. R., \& Álvares, A. C. M. (2019). Intoxicação medicamentosa relacionada à tentativa de autoextermínio. Rer. Inic. Cient. Ext., 2(2): 102-8.

Silva, H. C. G., \& Costa, J. B. (2018). Intoxicação exógena: casos no estado de Santa Catarina no período de 2011 a 2015. Arq. Catarin. Med. jul-set. 47(3):0215 .

Souto, C. E., Santos, M. O. A., Osela, M. E. G. B., \& Dutra, D. A. (2012). Intoxicações medicamentosas em Araucária - PR. Revista Uniandrade. 13 (3): $210-$ 220.

Ramos, T. O., Colli, V. C., \& Sanches, A. C. S. (2017). Indicadores epidemiológicos das intoxicações exógenas em crianças menores de 5 anos na região de Araçatuba-SP. Revinter, 10 (03): 86-100.

Rangel, N. L., \& Francelino, E. V. (2018). Caracterização do Perfil das Intoxicações Medicamentosas no Brasil, durante 2013 a 2016. Id onLine Rev. Mult. Psic. 12 (42): 121-135, ISSN 1981- 1179 .

Paula, T. C., Bochner, R., \& Montilla, D. E. R. (2012). Análise clínica e epidemiológica das internações hospitalares de idosos decorrentes de intoxicações e efeitos adversos de medicamentos, Brasil, de 2004 a 2008. Rev. Bras. Epidemiol., 15(4): 828-44.

Palhano, T. A., \& Herrerias, T. (2020). Avaliação do perfil de intoxicações medicamentosas nos anos de 2018 e 2019 no Brasil. Trabalho de conclusão de curso - TCC. Bacharelado em Farmácia. Guarapuava - PR. Disponível em: http://200.150.122.211:8080/jspui/handle/23102004/194

Teles, A. S., Oliveira, R. F. A., Coelho, T. C. B., Ribeiro, G. V., Mendes, W. M. L., \& Santos, P. N. P. (2013). Papel dos medicamentos nas intoxicações causadas por agentes químicos em município da Bahia, no período de 2007 a 2010. Rev. Ciênc. Farm. Básica Apl.,34(2):281-288, ISSN 1808-4532.

Viana, A. M. Neto., Ferreira, M. A. D., Figueiredo, S. M. F. B., Silva, F. M. B., Soares, A. C. S., \& Gondim, A. P. S. (2009). Aspectos Epidemiológicos da intoxicação por medicamentos em crianças e adolescentes atendidos no centro de assistência toxicológica do estado do Ceará. Revista Baiana, 33(3): 388-401.

Vieira, D. M., \& Caveião, C. (2016). Perfil das intoxicações medicamentosas no estado de São Paulo na perspectiva da vigilância sanitária. Revista Saúde e Desenvolvimento, 9(5):120,141.

Vinhal, D. C., \& Soares, V. H. C. (2018). Intoxicação por organofosforados: Uma revisão da literatura. Revista Científica FacMais, 14 (3): Out. ISSN 22388427. 